VS09-04

\section{Structure and mechanism of C-acyltransferase from pseudomonas protegens}

Tea Pavkov-Keller' ${ }^{1}$, Nina G. Schmidt², Anna Żądło-Dobrowolska ${ }^{3}$, Wolfgang Kroutil ${ }^{4}$, Karl Gruber ${ }^{5}$

1. University of Graz, Institute of Molecular Biosciences and ACIB, Graz, Austria

2. University of Graz, Institute of Chemistry and ACIB, Graz, Austria

3. University of Graz, Institute of Chemistry, Graz, Austria

4 .University of Graz, Institute of Chemistry and BioTechMed, Graz, Austria

5. University of Graz, Institute of Molecular Biosciences and BioTechMed, Graz, Austria

email: tea.pavkov@uni-graz.at

$\mathrm{C}-\mathrm{C}$ bond forming reactions are the key transformations to set up the carbon framework of organic molecules. In this sense, the Friedel-Crafts acylation is commonly used for the synthesis of aryl ketones, that are motifs in many fine chemicals and natural products. Although various types of catalysts have been described for this reaction, a biocatalytic version, which may benefit from the chemo- and regioselectivity of enzymes, has not yet been introduced. Here, we describe a bacterial multi-component C-acyltransferase from Pseudomonas protegens that can catalyze Friedel-Crafts $C$-acylation of phenolic substrates in buffer without the need of CoA-activated reagents reaching up to $>99 \%$ conversion $[1,2]$. We determined $\mathrm{x}$-ray crystal structures of the complex using diffraction data from two different crystal forms at 2.8 and $3.4 \AA$ resolution, respectively. This multimeric enzyme consists of three subunits - PhlA, PhlB and PhlC. They are arranged in a $\operatorname{Phl}\left(\mathrm{A}_{2} \mathrm{C}_{2}\right)_{2} \mathrm{~B}_{4}$ composition, in which the four copies of PhlB mediate the binding of two PhlA and two PhlC dimers to form the complete oligomer. Soaking the native donor/acceptor monoacetylphloroglucinol into crystals of ATase and solving the complex structure revealed together with mutations that only residues from PhlC interact with the substrate, which indicates that this is the subunit responsible for the transferase activity we observed.

References:

[1] Schmidt, N. G., Pavkov-Keller, T., Richter, N., Wiltschi, B., Gruber \& K., Kroutil, W. (2017). Angew Chem Int Ed Engl 56, 7615-7619.

[2] Żądło-Dobrowolska, A., Schmidt, N.G. \& Kroutil, W. (2018). Chem Commun 29, 3387-3390.

Keywords: acyltransferase, multi-enzyme complex, FriedelCrafts acylation

\section{MS09-05}

\section{Structural snapshots of multiple enzyme- ligand complexes pave the road for semi rational enzyme engineering}

Zsofia Bata ${ }^{1}$, Bence Molnár ${ }^{2}$, Ibolya Leveles ${ }^{2}$, Andrea Varga ${ }^{3}$, Csaba Paizs $^{3}$, László Poppe ${ }^{1}$, Beáta G. Vértessy²

1. Budapest University of Technology and Economics, Department of Organic Chemistry and Technology, Budapest, Hungary

2. Budapest University of Technology and Economics, Biostruct laboratory, Department of Applied Biotechnology and Food Science, Budapest, Hungary

3. Babeş-Bolyai University Faculty of Chemistry and Chemical Engineering, Cluj-Napoca, Romania

\section{email: bata.zsofia@mail.bme.hu}

Aromatic amino acid ammonia-lyases and 2,3-aminomutases contain the post-translationally formed prosthetic 3,5-dihydro-4-methylidene-5H-imidazol-5-one (MIO) group [1]. The so-called MIO-enzymes may be used for the stereoselective synthesis of enantiopure $\alpha$ - or $\beta$-amino acids, making these chemical processes more environmentally friendly and more affordable. Although, a number of structures are available in the PDB for MIO enzymes, the catalytically important Tyrloop [1] is either missing or is in a catalytically inactive conformation, due to the "loop-out" conformation in eukaryotic phenylalanine ammonia-lyase (PAL) structures (eg.: PDB:1W27 [2]). Lack of protein structure in a catalytically competent conformation impeded understanding the PAL mechanism and the key reasons for the enantioselectivity of the enzyme.

Our recent study characterized novel amino phosphonic acid inhibitors of MIO enzymes. [3] Enzyme kinetic measurements and isothermal titration calorimetry demonstrated that addition of a methylidene substituent to the $\beta$-carbon atom of the phosphonic acid analogue of the natural substrate, 1-Phe, enhanced binding $\left(\mathrm{K}_{\mathrm{d}}=40 \mathrm{nM}\right)$ and reversed the enantiopreference of the enzyme. [3] Importantly, inhibitors characterized in this study, also facilitated the crystallization of PcPAL. The resulting refined structure is shown on Figure 1. It confirms the N-MIO catalytic mechanism and demonstrates that the strong binding of (S)-APPA is due to a covalent-like hydrogen bond between its phosphonic acids group and the catalytic Tyr110.

Further key insights were gained, as the protein also crystallized in complex with cinnamic acid, the product of the natural reaction, and d-phenylalanine, the non-reactive enantiomer. In addition we could also employ sodiumborohydride to reduce the MIO group, thus render the enzyme catalytically inactive without significantly distorting the active site geometry. Such reduced enzyme crystallized from the same conditions as the native enzyme in the presence of the natural substrate 1-phenylalanine. Taken together these three new structures refine our understanding of the ligand binding modes and the catalytic mechanism of PcPAL. Moreover, analysis of the structures identified three key residue positions in the carboxyl-binding region that can be targeted for altering the enantioselectivity of the enzyme.

1. Figure: Electron densities and modeled structure of the active site of PcPAL in complex with covalently bound but still reversible inhibitor (S)-APPA[3]. Unequivocally identifying the geometry of the bound ligand and the covalent bond formed (1.4 $\AA$ ) with the MIO group. Resolution $1.9 \AA$. 